Widefield ScIENCE AND TeChNOLOGY For the SKA

SKADS CONFERENCE 2009

S.A. Torchinsky, A. van Ardenne, T. van den Brink-Havinga, A.J.J. van Es, A.J. Faulkner (eds.)

4-6 November 2009, Château de Limelette, Belgium

\title{
BEST: Basic Element for SKA Training ${ }^{\star}$
}

\author{
S. Montebugnoli ${ }^{1}$, G. Bianchi ${ }^{1}$, J. Monari ${ }^{1}$, G. Naldi ${ }^{2}$, F. Perini ${ }^{1}$, and M. Schiaffino ${ }^{1}$ \\ 1 I.N.A.F. - I.R.A, Via Fiorentina, 3508/B 40059 Medicina, Italy \\ email: s.montebugnoli@ira.inaf.it, g.bianchi@ira.inaf.it, j.monari@ira.inaf.it,f.perini@ira.inaf.it, \\ mschiaffino@med.ira.inaf.it \\ 2 Università degli Studi di Bologna, Dipartimento di Astronomia, Via Ranzani 1, 40127 Bologna, Italy \\ email: gnaldi@med.ira.inaf.it
}

\begin{abstract}
In the frame of the SKADS activities, efforts have been devoted to the design of a test bed (BEST-Basic Element for SKA Training), composed of low cost, high performance receivers, optical links and a fast low power consumptive modular back end. Such a prototype is characterised by a geometrical collecting area of about $1400 \mathrm{~m}^{2}$ and exploits some cylindrical concentrators, mechanically modified, of the large Northern Cross array. These modifications were required in order to host both the new front ends on the focal lines and the optical links (to transport the analogue signals to the processing room) on the antenna structures. The architecture chosen for the BEST pathfinders is a standard single conversion receiver. A low frequency $(120-240 \mathrm{MHz})$ prototype has been prepared and named BEST-31o. Some preliminary astronomical observation tests on CAS-A was performed with promising results with both the UHF(BEST-2) and VHF(BEST-3lo) prototypes.
\end{abstract}

\section{Introduction}

In order to fulfill the requirements of the description of Work (DoW) of SKADS (Montebugnoli et al. 2007), different in size pathfinders, based on cylindrical concentrators of the existing Northern Cross radio telescope, have been designed and named BEST (Basic Element for SKA Training) 1, 2 and 3lo (low frequency).

BEST-1: it was a first level prototype $\left(170 \mathrm{~m}^{2}\right)$ equipped with 4 front-ends directly installed on the focal line of a single N/S arm cylindrical concentrator (about $24 \times 7.5 \mathrm{~m}$ ) and with the IF stages in the receiver room. It allowed to test the new low cost and high performance electronics and to start understanding some concepts in order to better define the following BEST2 test bed architecture.

BEST-2: it is composed of 32 single conversion receivers, the front-ends are installed on 8 cylindrical concentrators $\left(1440 \mathrm{~m}^{2}\right)$ as visible in Fig. 1, with a $16 \mathrm{MHz}$ wide IF channel centred at $30 \mathrm{MHz}$. It is very useful to focus on the low cost high performance hardware developed for BEST-1, the design of data transportation via optical fibre and configuration of an FX correlator. The correlator is based on the IBOBs and BEE2 boards from the CASPER group (University of Berkeley, USA).

BEST-31o: it is a $800 \mathrm{~m}^{2}$ prototype that represents a technological bridge from SKADS (FP-6) to the next step PrepSKA (FP-7). It operates in the 120-240 MHz band and will give a lot of experience and technology for the AA-lo (Aperture Arraylow frequency).

Before to describe the block diagrams, let us summarize some reliability prediction considerations on the electronic chain.

\footnotetext{
^ This work was supported by the European Commission Framework Program 6, Project SKADS, Square Kilometre Array Design Studies (SKADS), contract no 011938.
}

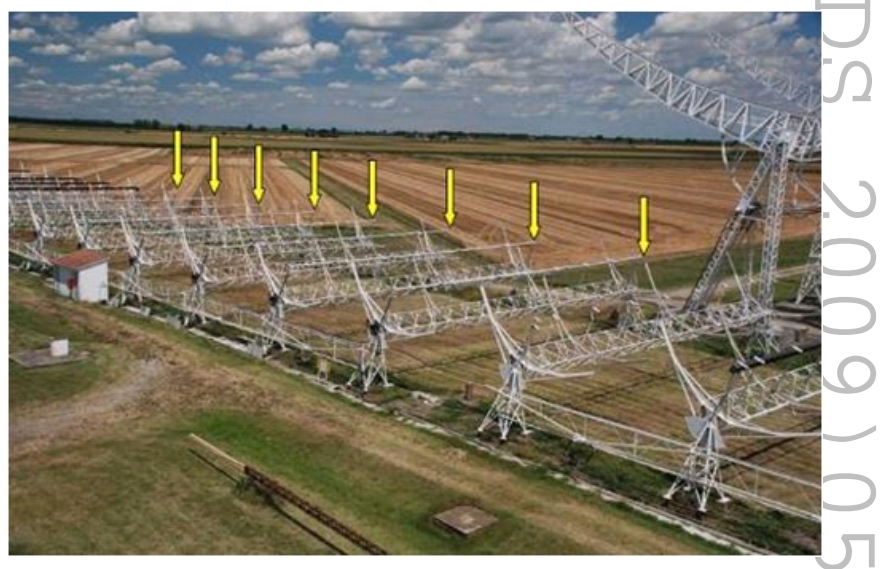

Fig. 1: Four receivers on 8 different cylindrical concentrators.

\section{Reliability}

Once the block diagram of a receiver is clearly conceived, it is necessary to perform a reliability investigation before to deal with the construction (Montebugnoli et al. 2006). This is important to place each block of the chain in the more suitable environment (Ground Fixed Uncontrolled, Ground Benign Controlled etc..) in order to increase the MTBF (Mean Time Between Failure). To emphasize such a concept a comparison in terms of MTBF between two different solutions (same block diagram but different displacements of the blocks), are here following reported:

- RF transported with an analogue optical link directly from the front end to a protected room (Fig. 2),

- RF transported with cable to the A/D in the cabins and then transported with digital optical link to the processing room (Fig. 3). 


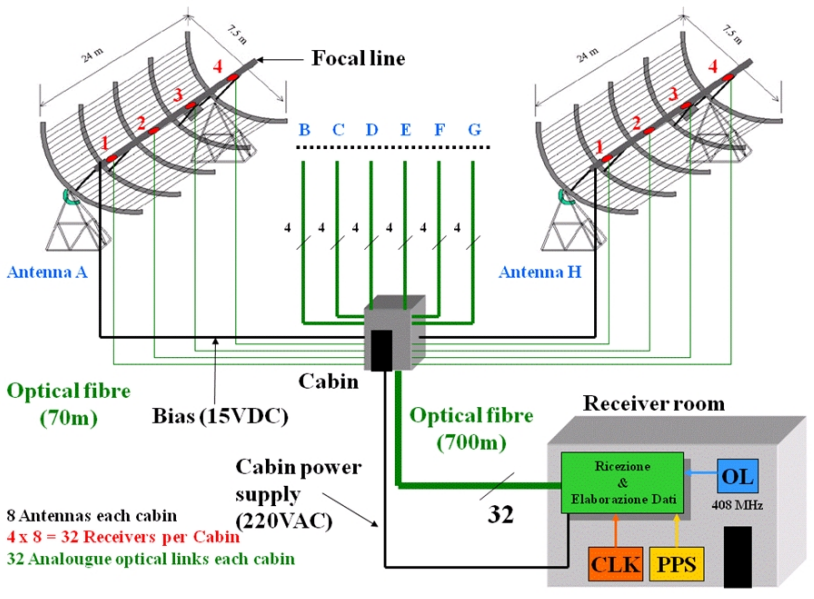

Fig. 2: RF transported with an analogue optical link from the front end directly to a protected room.

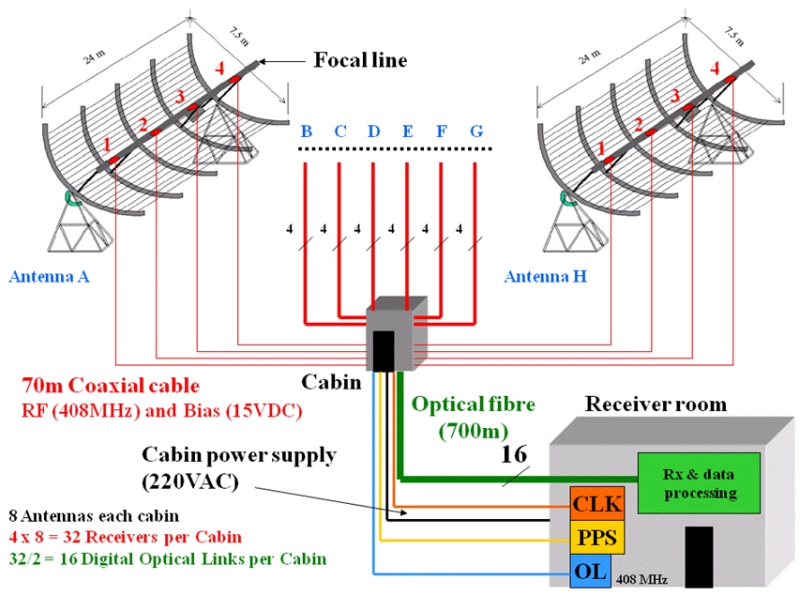

Fig. 3: RF transported with cable to the A/D in the cabins and then transported with digital optical link to the processing room.

This analysis has been made according to the data-base MILHDBK-217-FN2 (Mode I case 3), assuming an operative temperature of $30{ }^{\circ} \mathrm{C}$ and a $100 \%$ Duty Cycle (24/24 hours), independent faults and constant failure rate.

Then, different operative environments have been considered: the GM (Ground Mobile) for the antenna, GF (Ground Fixed Uncontrolled) for the cabin and GB (Ground Benign Controlled) for the processing room. From the reliability analysis, the digital link option presents a MTBF of about 10624 hours (about 1.2 years without any maintenance). Under the same hypothesis, the analogue link option exhibits a $\mathrm{MTBF}=37187$ hours, that correspond to about 4.2 years. The analogue link solution increases the reliability of the system because the major part of the processing hardware is indoor (in a temperature and humidity controlled room). The system MTBF trend in the two possible solutions (Vs temperature) is visible in the Fig. 4). A suitable solution to increase the MTBF was to transport the RF directly from the front end (GM environment - Ground Mobile) to a protected room via an analogue

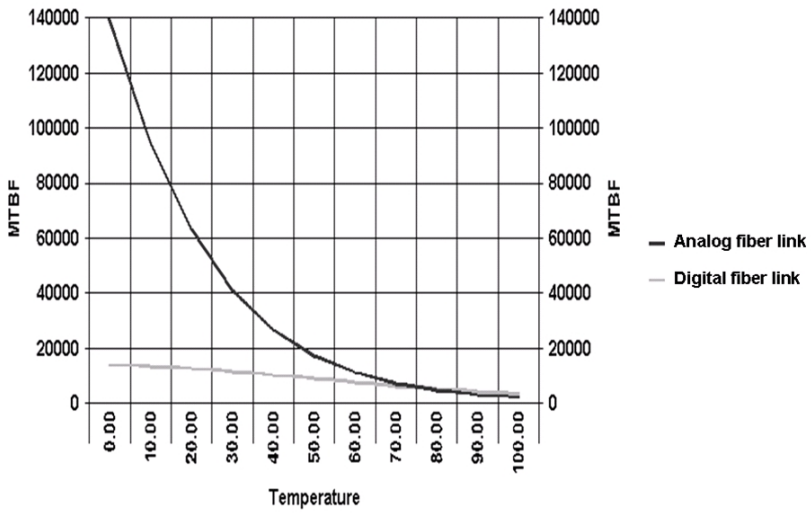

Fig. 4: Analogue and digital optical link system MTBF vs Temperature.

optical link as reported in Fig. 2 (Catelani et al. 2006). The architecture of the BEST pathfinder is a standard single conversion receiver as sketched in Fig. 5. The front ends are installed on the focal lines and the RF signals are directly transported to the receiver room via an analogue optical link. After a down conversion at $30 \mathrm{MHz}$, they are digitised (with the sufficient number of bits for the required dynamic range) and then processed. The IF boards provide an extra output at the RF level $(408 \mathrm{MHz})$ as well. This allows to perform quick tests on the RF part of the receiver chain (i.e. signal level, RFI monitoring, Front End or optical TX fault). A direct RF output gives also the opportunity to implement some tests on the direct RF sam pling. Here following there is the description of the electronics of each blocks.

\section{BEST-1\&2}

\subsection{Front end}

Different LNA architectures have been investigated. The chosen configuration was the balanced one for its good impedance matching, high dynamic range, reasonable low noise and low cost (Fig. 6 and Fig. 7).

\subsection{Optical link}

The analogue optical link used to transport the RF from the front end to the receiving room has been obtained in the frame of a collaboration between INAF and the ANDREW WIRELESS SYSTEMS local factory. The features of the ANDREW custom optical link are reported in Table 1 (Catelani et al. 2009b). The good phase and amplitude stability of the links demonstrated the possibility to calibrate and operate with the BEST-2 array.

\subsection{IF stage}

Due to reliability prediction considerations, the IF stages have been installed inside a temperature controlled room and connected to the front end via an analogue optical link. All the IF stages are installed in some 19" rack cabinets along with 


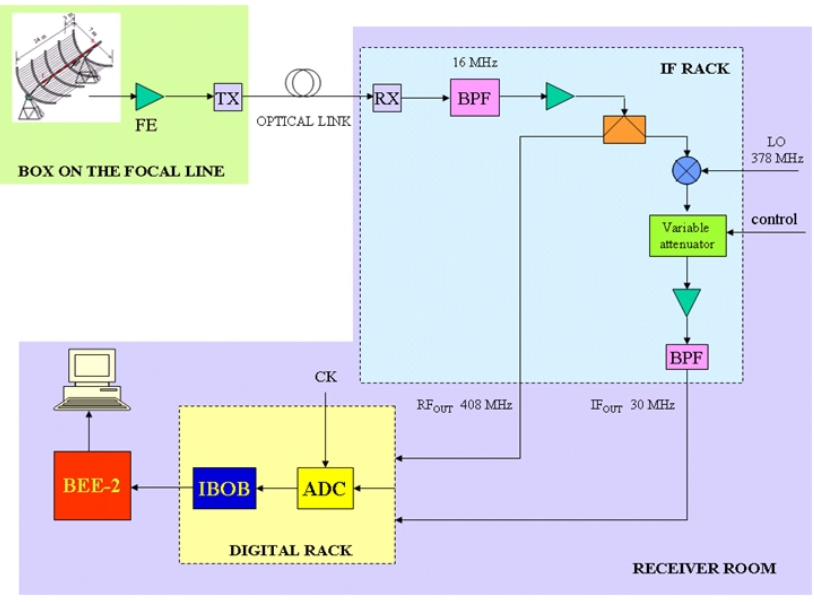

Fig. 5: Block diagram of the receiver chain.

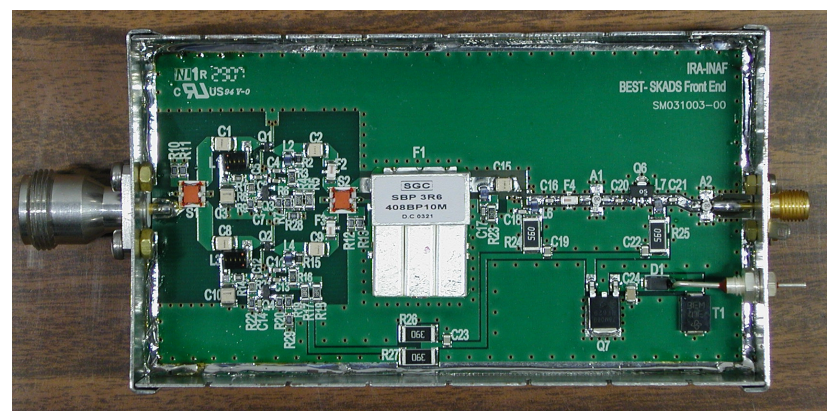

Fig. 6: BEST-2 Front End.

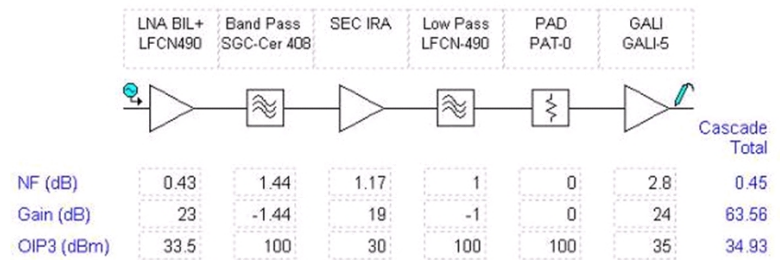

Fig. 7: Front end main features.

the Sync and LO distributors, clock generator, H-Maser locked synthesizer, fast data acquisition and post processing block. The system is composed of individual rack mounted modules for easy replacement (Fig. 8).

\subsection{LO and Sync distribution}

A Xmas tree configuration has been selected in order to assure the same phase of the LO to each mixer. The same philosophy was implemented for the sync distribution. These are crucial issues to accomplish the task in large array.

\section{BEST-3lo}

In order to set up a technological bridge between SKADS and PrepSKA-AAlo, it was decided to exploit a $24 \mathrm{~m}$ segment of
Table 1: ANDREW custom optical link features.

\begin{tabular}{|l|c|c|}
\hline \hline \multicolumn{3}{|c|}{$\begin{array}{c}\text { TFTY2324w+TFRY501 } \\
100-1000 \mathrm{MHz}\end{array}$} \\
\hline Gain & {$[\mathrm{dB}]$} & -25 \\
\hline Gain flatness & {$[\mathrm{dB}]$} & \pm 0.75 \\
\hline Input Return Loss & {$[\mathrm{dB}]$} & $>15$ \\
\hline Output Return Loss & {$[\mathrm{dB}]$} & $>20$ \\
\hline Input IP3 & {$[\mathrm{dBm}]$} & +33 \\
\hline Noise Figure & {$[\mathrm{dB}]$} & 36 \\
\hline
\end{tabular}

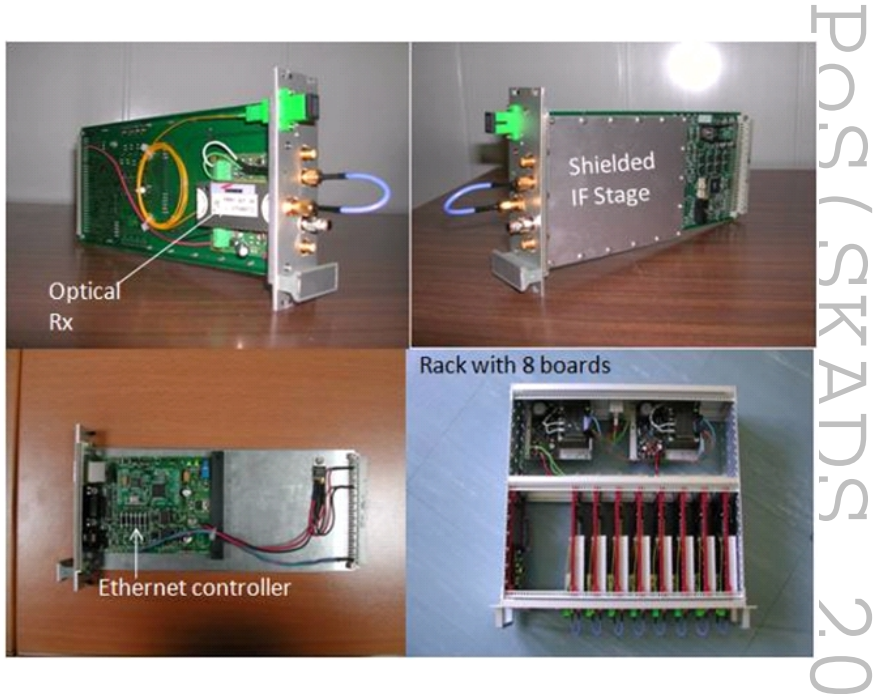

Fig. 8: Different views of the IF board and 8 boards already assembled in a 19 " rack.

the E/W cylindrical concentrator of the Northern cross antenna as a low frequency pathfinder in the $120-240 \mathrm{MHz}$ frequency band with approximately $800 \mathrm{~m}^{2}$ of collecting area. In order to cover the $120-240 \mathrm{MHz}$ frequency band, an electromagnetic investigation of a Log Periodic Antenna (LPA) able to illuminate the main reflector with good efficiency, has been done.

The rough schematic block diagram of the low frequency BEST (embryonic AAlo prototype) is reported in Fig. 9: a pass band filter bank in the front end allows to select the more "clean" VHF band slice in order to easily perform the preliminary calibration tests. The RF signal is transported with an analogue optical link to the processing room and then directly digitized. In Fig. 10 is reported a picture of the above mentioned log-periodic antennas already installed on the $\mathrm{E} / \mathrm{W}$ arm focal line.

\section{Data acquisition}

In order to exploit the best flexibility a modular programmable data processing system, developed by the CASPER group of the University of Berkeley, has been adopted. This system is composed of a 1 GSPS dual A/D converter for each receiver connected to a serialiser board (iBOB) that can host up to 4 A/D inputs. These boards are connected to the FPGA cluster Bee2 board via high speed InfiniBand CX4 links, Fig. 11 and Fig. 12. 


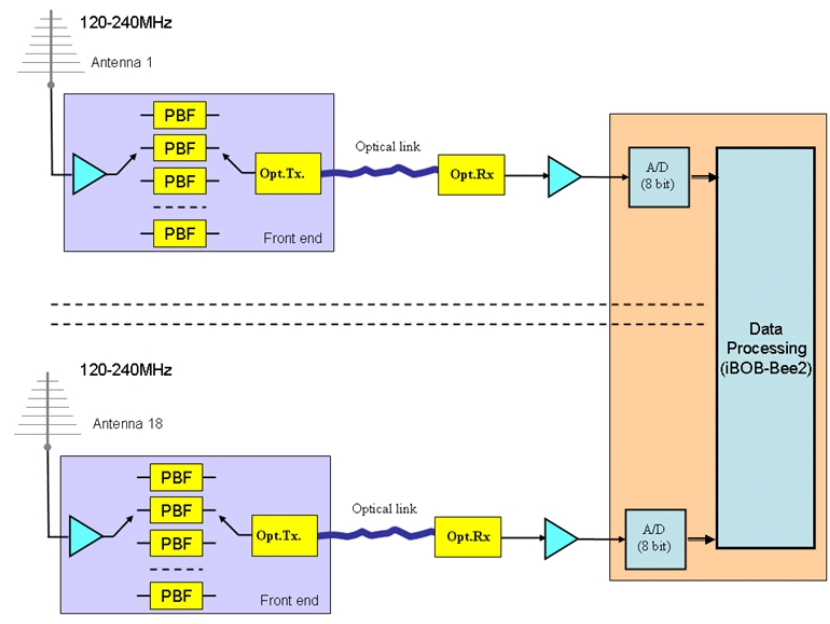

Fig. 9: Schematic block diagram of the Low Frequency BEST (embryonic AAlo prototype).

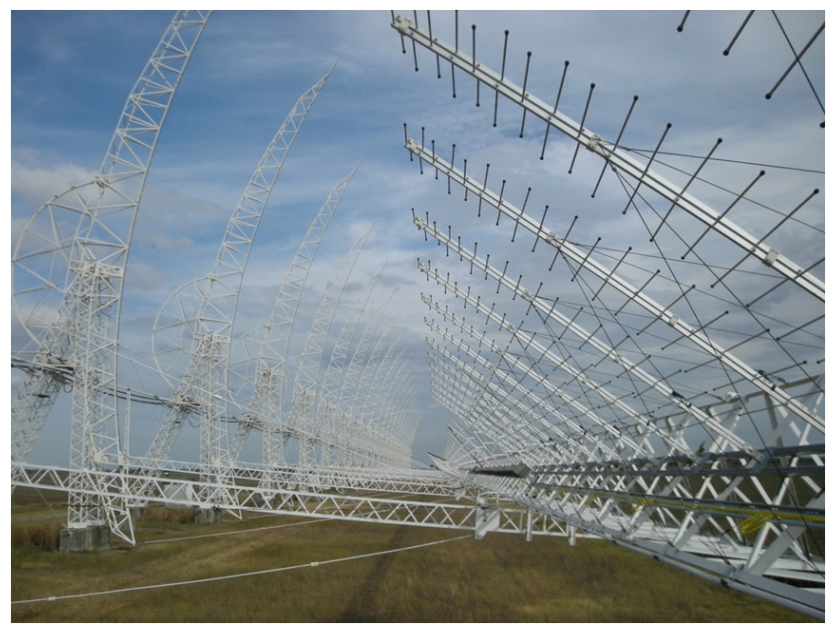

Fig. 10: Low frequency array installed on one sector of the E/W focal line.

The A/D converters operate with 8 bits that means about 48 $\mathrm{dB}$ of dynamic range. In this case an FX correlator (Parsons et al. 2008) is required since such a configuration saves approximately 4 orders of magnitude of operations compared to an $\mathrm{XF}$ correlator and, in addition, it allows mitigation of RFI signals switching off the interfered channels at the polyphase filter bank level. In the case of the BEST-2, a 32 receivers and 1024 channels FX correlator has been designed. The data acquisition and processing is shared by the BEST-2 and BEST-3lo prototypes (Fig. 13). In this way the data acquisition is a common block for both the prototypes. At present the configuration of the Bee2 FPGA cluster offers approximately $500 \mathrm{GOp} / \mathrm{sec}$ with a $400 \mathrm{~W}$ power supply $(1.2 \mathrm{GOps} / \mathrm{W})$. This provides enough computation power to implement a full 32 stations correlator for the BEST-2 demonstrator and a full beamformer $(4 \mathrm{MHz})$ for the 16 antennas of the BEST-3lo system.

A first light (CAS-A) of BEST-2 was obtained late 2007, as visible in Fig. 14, while the first map (CAS-A) was available late 2008 (Fig. 15). The preliminary test to see the fringes (on

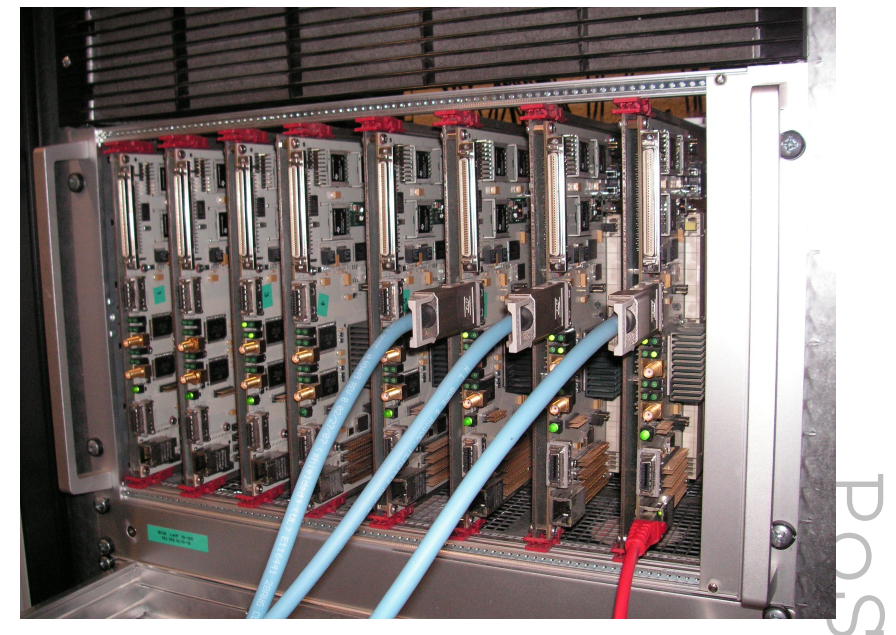

Fig. 11: ADCs+iBOB.

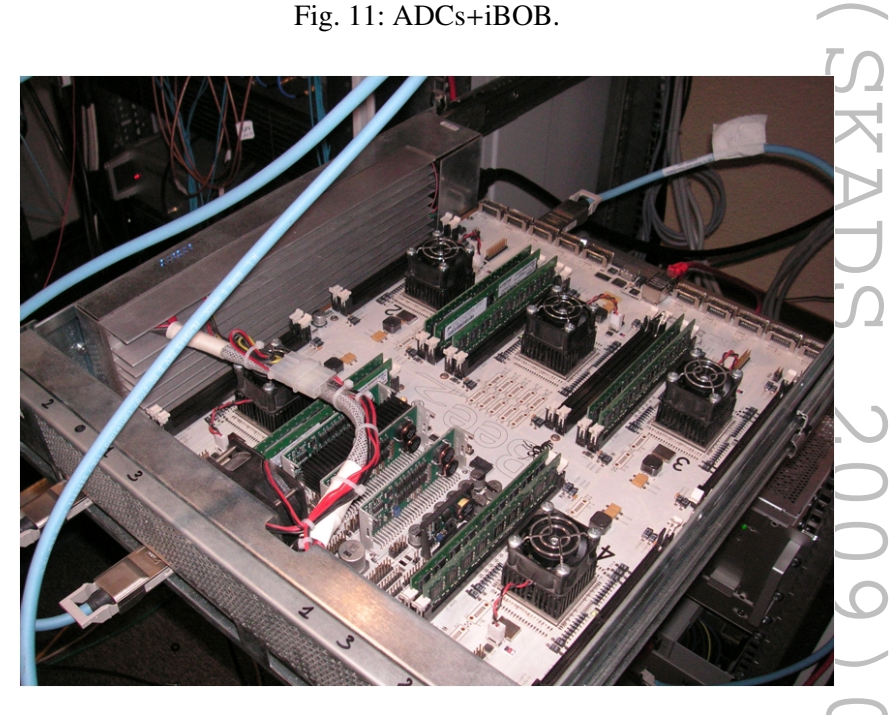

Fig. 12: Bee2 board.

the transit of the sun) with the BEST-3lo, in the 138-142 MHz band, is reported in Fig. 16. In this figure it is clear that the strong RFI are sometimes switched off and then on again. An important result is that the dynamic range in both the analogue and digital domain seems to be sufficient.

Finally, a MVDR adaptive beamforming algorithm has been successfully simulated and the implementation on the IBOB boards is in progress. A survey of possible RFI mitigation algorithms is reported in Table 2.

\section{Conclusions}

The BEST-1 and BEST-2 prototypes based on cylindrical concentrators have played a fundamental role to understand how to handle the different issues concerned with the construction of large array of sensors. These test beds allowed the first astronomical observations of CAS-A as well. The BEST-3lo (120-240 MHz) allowed to set up a low frequency technological bridge from SKADS to PrepSKA (AA-lo). A first fringes observation of the sun were presented to underline the very 


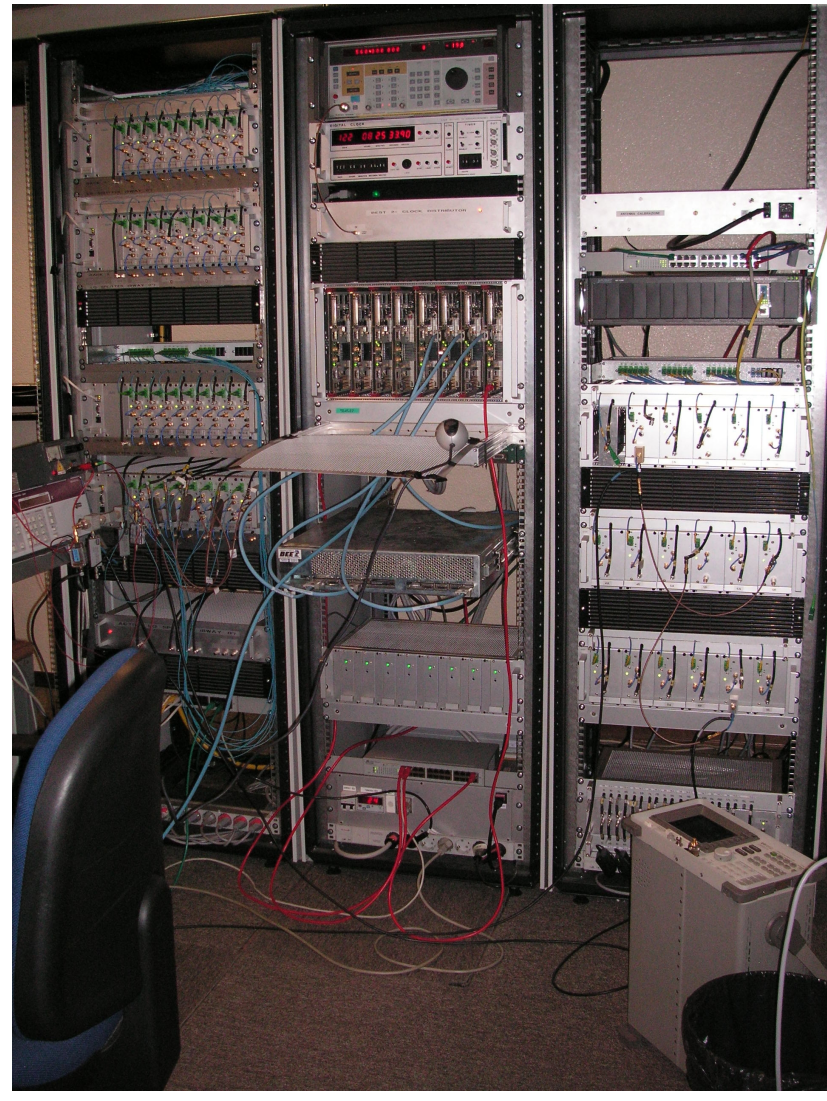

Fig. 13: BEST-2 (left), BEST-3lo (right) and the IBOBs and Bee2 data acquisition and processing (center).

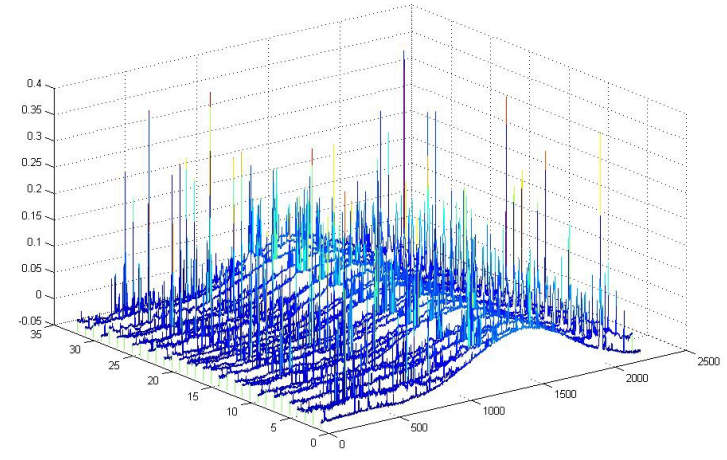

Fig. 14: BEST-2 first light - Total power (September 2009).

good dynamic performances in both the analogue and digital domain.

\section{References}

Catelani, M., V. L. Scarano, I. Trotta, R. Singuaroli, S. Montebugnoli, G. Bianchi, "Reliability tests and experimental analysis on radioreceiver chains," IEEE Instrumentation and Measurement Technology Conference, Sorrento (Italy),

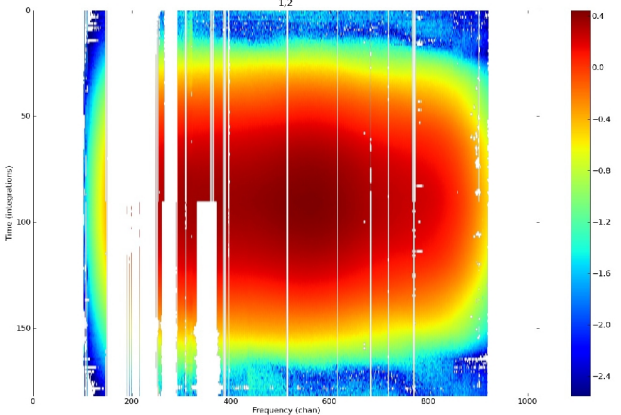

Fig. 15: BEST-2 first radio map with RFI blanked, CAS-A (September 2008).

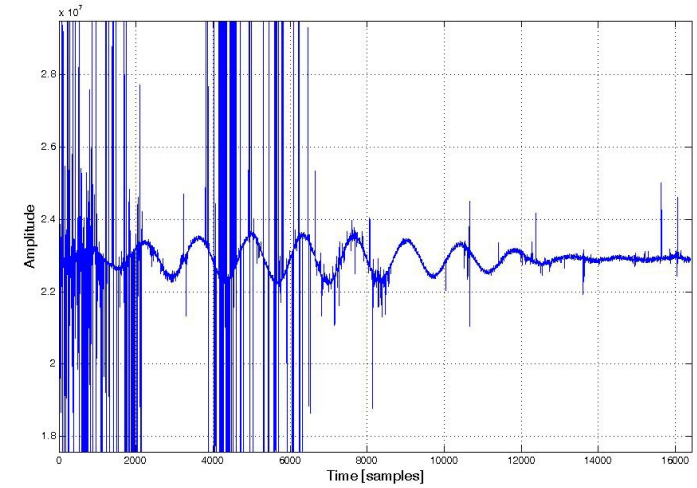

Fig. 16: Preliminary very dirty fringes (Sun transit) with two of $16 \log$ periodic antennas of BEST-3lo (November 2009).

Table 2

\begin{tabular}{|l|c|c|c|}
\hline \hline Method & $\begin{array}{c}\text { Implementation } \\
\text { time required } \\
\text { (3 GHz clock PC) }\end{array}$ & $\begin{array}{c}\text { Applicability } \\
\text { in a static } \\
\text { environment }\end{array}$ & $\begin{array}{c}\text { Applicability } \\
\text { in a dynamic } \\
\text { environment }\end{array}$ \\
\hline MVDR & $9.313 \mathrm{sec}$ & Good & Good \\
\hline $\begin{array}{l}\text { FD- } \\
\text { LCMV }\end{array}$ & $54.53 \mathrm{sec}$ & Very good & $\begin{array}{c}\text { Not } \\
\text { applicable }\end{array}$ \\
\hline $\begin{array}{l}\text { Null } \\
\text { steering }\end{array}$ & $9.125 \mathrm{sec}$ & Good & Good \\
\hline KLT & $9.031 \mathrm{sec}$ & Good & Good \\
\hline
\end{tabular}

April 24-27, 2006

Catelani, M., V. L. Scarano, R. Singuaroli, S. Montebugnoli, G. Bianchi, F. Perini, "Environmental Testing: Behavior of a Radio receiver Chain Front-end," Metrology and Measurement Systems VOLUME XVI - NUMBER 1/2009

Catelani, M., V. L. Scarano, R. Singuaroli, I. Trotta, S. Montebugnoli, G. Bianchi, F. Perini, "Northern Cross Radiotelescope: test and measurements of reliability performance on radioreceiver chains," IEEE Transactions on Instrumentation and Measurement, Vol.58, Issue 10, October 
2009

Montebugnoli, S., G.Bianchi, F. Perini, C. Bortolotti, A.Cattani, A. Maccaferri, A. Cremonini, M. Roma, J.Roda, G. Zacchiroli, Italian "SKA test bed based on cylindrical antennas," Astron. Nachr./AN327, No.5/6, 624-625 (2006) /DOI 10.1002/asna.2006 10606

Montebugnoli, S., G. Bianchi, J. Monari, F. Perini, M. Schiaffino, G.P. Zacchiroli and G. Naldi, "Square kilometre Array (SKA): il radiotelescopio di prossima generazione e tecniche di multibeaming," MECSA Salerno (I) 14/15 Maggio 2007

Parsons, A., D. Backer, H. Chen, P. Droz, T. Filiba, D. MacMahon, J. Manley, P. McMahon, A. Parsa, D. Werthimer, M. Wrigh, "A Scalable Correlator Architecture Based on Modular FPGA Hardware and Data Packetization," Submitted for publication to IEEE transaction on Signal Processing, February 4, 2008 